\section{Incidence of symptoms in previously symptom-free impacted lower third molars assessed in general dental practice}

\author{
M. J. Fernandes, ${ }^{1}$ G. R. Ogden, ${ }^{2}$ N. B. Pitts, ${ }^{3}$ S. A. Ogston ${ }^{4}$ and D. A. Ruta ${ }^{5}$
}

IN BRIEF

- Sheds more light onto the natural history of impacted lower third molars.

- Correlates the development of symptoms with some clinical characteristics.

- Provides the general dental practitioner with more knowledge that could help when deciding whether or not to extract an impacted third molar.

- Encourages debate in relation to current practice within the United Kingdom.

\begin{abstract}
Aim To determine the potential of a pathology-free impacted lower third molar to cause symptoms within a year and whether these symptoms can be linked to clinical characteristics, lifestyle or socio-demographic status. Design One-year prospective cohort study of patients registered in general dental practice in Scotland with at least one asymptomatic impacted lower third molar. Methods All general dental practices with panoramic radiography facilities in Tayside, Fife and Greater Glasgow (Scotland, UK) were invited to participate in the study. Orthopantomographs taken between 1995 and 2002 were reviewed and eligible patients were contacted and invited to participate. Patients were assessed in their own dental surgery by the same research dentist. In this baseline assessment, the presence of impaction was confirmed and all patients with a previous history of symptoms and/or pathology were excluded from further analysis. Clinical characteristics such as the angulation and the degree of impaction were recorded. Patients also completed a socioeconomic questionnaire. Eligible patients were re-assessed by the same research dentist one year later when they were asked about their experience of symptoms within the past year. Information was cross-referenced with patients' dental records. Results A total of 613 patients attended the baseline appointment. Of those, 30 (4.89\%) had a history of symptoms and were excluded from the study, leaving 583 (95.10\%) eligible patients. From those, 421 (69\%) patients with a total of 676 lower third molars were examined one year later. 22.67\% of all vertically impacted teeth examined had developed symptoms, along with $13.15 \%$ of all mesially impacted, $30.69 \%$ of all distally impacted and 6.45\% of all horizontal third molars. This association was statistically significant ( $p$ <0.001). 23.05\% of all partially erupted teeth and a surprising $10.49 \%$ of all unerupted teeth were associated with symptoms during the study period. This association was also significant $(p \leq 0.001)$. There was also a statistically significant inverse association between the development of symptoms and age $(p=0.0028)$. Conclusions The predictability that an impacted lower third molar will develop symptoms in future remains unclear. However, some clinical characteristics such as the angulation, the degree of impaction and the patient's age could be useful in predicting the likelihood of future symptomatology.
\end{abstract}

\section{INTRODUCTION}

In the past decade there has been much discussion on the management of impacted wisdom teeth. In the 1990s the cost of third molar surgery to the NHS was estimated to be approximately £30

\footnotetext{
1*General Dental Practitioner, 19 Rubislaw Terrace, Aberdeen, AB10 1XE; ${ }^{2}$ Unit of Oral Surgery and Medicine, University of Dundee Dental School, Park Place, Dundee, DD1 4HN; ${ }^{3}$ Dental Health Services Research Unit, ${ }^{4}$ Section of Public Health, Centre for Primary Care \&t Population Research, University of Dundee, Kirsty Semple Way, Dundee, DD2 4BF; ${ }^{5}$ nstitute of Health and Society, Newcastle University, Newcastle upon Tyne, NE1 7RU

${ }^{*}$ Correspondence to: Dr Marcelo Fernandes Email:mfernandes10@hotmail.com
}

\section{Online article number E10}

Refereed Paper - accepted 10 July 2009

DOI: 10.1038/sj.bdj.2009.804

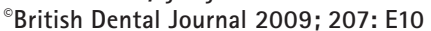

million per annum ${ }^{1}$ and around £22 million to the private sector. ${ }^{2}$ The enormous number of teeth removed in the UK prompted researchers to assess the type of practice conducted here and in 1998 a study had estimated that around 20-30\% of third molars that were removed in the UK did not fulfil the American National Institutes of Health criteria for removal and were, therefore, removed prophylactically. ${ }^{3}$ This was followed by the publication of guidelines by the $\mathrm{NICE}^{4}$ and $\mathrm{SIGN}^{5}$ on the management of impacted lower third molars and, possibly as a result, the number of impacted wisdom teeth removed in the UK started to fall. In addition, it is believed that changes in the legislation on the prescription of general anaesthesia have also resulted in a reduction in surgical extractions of impacted third molars. ${ }^{6}$

Are we right to be removing fewer third molars? Although the current literature on wisdom teeth is vast, the predictability that an impacted and pathology-free third molar will go on to develop disease is still uncertain. In 2005 a Cochrane Oral Health Group systematic review found no evidence to support or to refute the prophylactic removal of impacted wisdom teeth. ${ }^{7}$ The main clinically relevant questions are: 'What percentage of pathologyfree impacted third molars will remain free of disease if retained?' and 'How can we predict the likelihood of development of disease in these teeth?'

There is a lack of reliable evidence for the predictability that an unerupted and 
pathology-free third molar will cause disease. $^{8,9}$ A clinician's decision, therefore, has to be based on clinical and radiographic factors and also on the clinician's own perception and experience. Absolute indications and contra-indications for the removal of asymptomatic third molars cannot be established because there are no long-term studies on this subject at the present. ${ }^{8}$ Data that could help the clinician to decide what is the best treatment for a specific patient or case would be of great value.

Most recent publications on the management of impacted wisdom teeth favour non-intervention over extraction and although this approach seems to be the most reasonable, there are no conclusive studies to prove or disprove it. The aim of this study was to determine the potential of a pathology-free impacted lower third molar to be associated with symptoms and whether these symptoms can be linked to a particular clinical characteristic, lifestyle or socio-demographic status.

\section{METHODS}

The study was conducted entirely in the primary care setting. Three centres were involved: Tayside, Fife, and Greater Glasgow, Scotland, UK, with a total of 14 dental surgeries. The study received approval from respective research ethics committees in the centres studied. Patients were invited by a letter and/or a telephone call to attend an appointment in their own dental surgery where they would be seen by the 'wisdom tooth research dentist'. They also received a patient information leaflet with more details about the study.

To avoid exposing patients to irradiation it was decided that the best approach would be to recruit patients with impacted wisdom teeth who had already had a panoramic radiograph taken. Of course, this can generate selection bias and as a measure to decrease the probability of this type of bias, all patients with a history of problems with their lower third molars were excluded. The inclusion criteria for the study were:

- To be a registered patient within the dental primary care system of one of the three regions involved

- To be aged 18 to 70 years

- To have at least one impacted lower third molar

Table 1 Clinical characteristics of 613 subjects attending the baseline appointment according to gender and age group

\begin{tabular}{|l|l|l|l|l|l|l|}
\multirow{2}{*}{$\begin{array}{l}\text { Clinical } \\
\text { characteristics }\end{array}$} & \multicolumn{2}{|l|}{ Males } & Age group & Age group & Age group & Age group \\
$\mathbf{1}^{*}$ & $\mathbf{2}^{* *}$ & $\mathbf{3}^{* * *}$ & Age group & $\begin{array}{l}\text { Age group } \\
\mathbf{2}^{* * * *}\end{array}$ \\
\hline $\begin{array}{l}\text { Mean number } \\
\text { of teeth }\end{array}$ & 26.22 & 26.17 & 20.14 & 26.22 & 25.85 & 23.33 \\
\hline Mean max BPE & 2.09 & 2.61 & 2.93 & 1.65 & 2.03 & 2.78 \\
\hline $\begin{array}{l}\text { Mean Gingival } \\
\text { Bleeding Index }\end{array}$ & $54.52 \%$ & $47.05 \%$ & $26.02 \%$ & $38.41 \%$ & $44.79 \%$ & $24.07 \%$ \\
\hline $\begin{array}{l}\text { Mean Oral } \\
\text { Hygiene Index }\end{array}$ & 1.67 & 1.28 & 2.41 & 1.43 & 1.22 & 2.03 \\
\hline
\end{tabular}

Angulation of impaction:

\begin{tabular}{l|l|l|l|l|l|l}
\hline Mesial & $49.5 \%$ & $40.0 \%$ & $11.1 \%$ & $47.3 \%$ & $31.1 \%$ & $57.1 \%$ \\
\hline Vertical & $19.0 \%$ & $16.7 \%$ & $27.8 \%$ & $30.7 \%$ & $22.2 \%$ & $14.3 \%$ \\
\hline Distal & $13.5 \%$ & $13.3 \%$ & $38.9 \%$ & $16.0 \%$ & $26.7 \%$ & $14.3 \%$ \\
\hline Horizontal & $18.0 \%$ & $30.0 \%$ & $22.2 \%$ & $05.0 \%$ & $20.0 \%$ & $14.3 \%$
\end{tabular}

Degree of impaction:

\begin{tabular}{l|l|l|l|l|l|l}
\hline Partially erupted & $80.6 \%$ & $30.8 \%$ & $55.6 \%$ & $71.2 \%$ & $50.0 \%$ & $16.7 \%$ \\
\hline Unerupted & $19.4 \%$ & $69.2 \%$ & $44.4 \%$ & $28.8 \%$ & $50.0 \%$ & $83.3 \%$ \\
\hline
\end{tabular}

- To have had a panoramic radiograph taken between 1995 and 2002

- To have no current or past symptoms associated with impaction of third molars.

The baseline examination was conducted by a research dentist assisted by a dental nurse. All patients at baseline and at follow-up were assessed by the same dentist and the research dentist was calibrated externally against an oral surgeon also involved in the study. The angulation of the impaction was classified into mesioangular, disto-angular, vertical or horizontal based on the radiograph avaialble ${ }^{10}$ and into partially erupted or unerupted according to the communication with the oral cavity, where a periodontal probe was used in the distal of the lower second molar to establish communication in cases where it was not obvious. Patients reporting symptoms or presenting pathology associated with their impacted wisdom teeth were excluded from further analysis.

The other variables noted were the average number of teeth, a basic periodontal examination (BPE), ${ }^{11}$ gingival bleeding as measured by the Marginal Bleeding Index $(\mathrm{MBI})^{12}$ and the presence of plaque (PI) measured by the Oral Hygiene Index, ${ }^{13}$ gender, age and postcode (in order to obtain deprivation category as proposed by Carstairs and Morris). ${ }^{14}$ These clinical variables are summarised in Table 1 . Patients also completed a questionnaire which asked questions relating to education, employment, frequency of brushing, use of mouthwashes, tooth flossing, frequency of attending dental check-ups, time of last dental appointment, reason for last dental appointment, smoking and alcohol intake.

This population of patients with symptom-free impacted lower third molars was re-assessed one year later. In the followup appointment the presence of impacted lower tooth (or teeth) studied was noted and patients were asked about symptoms. Symptoms observed were pain and infection (or history of past infection) and caries in the third molar or visibly detectable caries in the distal of the adjacent tooth. It is worth noting that although clinical caries is not a symptom but a sign, we still incorporated it in the 'symptoms' category as this is the only sign of pathology investigated in this study and the development of signs could only be measured by taking new radiographs, which would not be justifiable or accepted by an ethics committee. Questions included were: 'Have you had 
any pain coming from your wisdom tooth in the past year?', 'Have you had any discomfort coming from your wisdom tooth in the past year?', 'Did you come to see your dentist in the past year due to problems with your wisdom tooth?', 'Has you dentist prescribed any antibiotics to you because of problems with your wisdom tooth in the past year?' and 'How many times did you have this problem in the past year?'. Patients reporting pain were asked to rate the pain on a 0-10 scale. Symptoms were defined as mild pain (scale 1-5), severe pain (scale 6-10), infection (pain + prescription of antibiotics/and or irrigation), discomfort/irritation and food stagnation. Information was cross-checked with GDPs' notes and in those few cases where there was a record of symptoms, patients were reminded of particular episodes.

It is imperative to note that not all symptoms included in this study were outlined as a reason for extraction according to the SIGN guidelines. ${ }^{5}$ These symptoms were, however, capable of causing an adverse reaction to patients and a decrease in one's oral health-related quality of life. ${ }^{15}$

Pearson chi-square tests were used to test the hypothesis of association between clinical characteristics of impaction and the reported development of symptoms. All variables presented in the socioeconomic questionnaire were analysed using $t$-tests to test for differences between patients who reported symptoms within the study period and those who were asymptomatic. We used SPSS 16.0 for all statistical calculations.

\section{RESULTS}

Six hundred and thirteen patients were recruited to the baseline appointment and out of those, 30 (4.89\%) were excluded for having a history of symptoms arising from their impacted lower third molar(s), leaving a total of 583 patients who were included in the study. In the one-year follow-up 32 patients were not contactable, leaving 551 patients who again were invited to attend for a follow-up examination.

The final number of patients examined at the one-year follow-up was 421 (69\% of all patients examined at baseline) resulting in 676 lower impacted third molars examined at baseline and again at the one-year follow-up. Out of those, 562 teeth (83.13\%) survived the study period symptom-free.

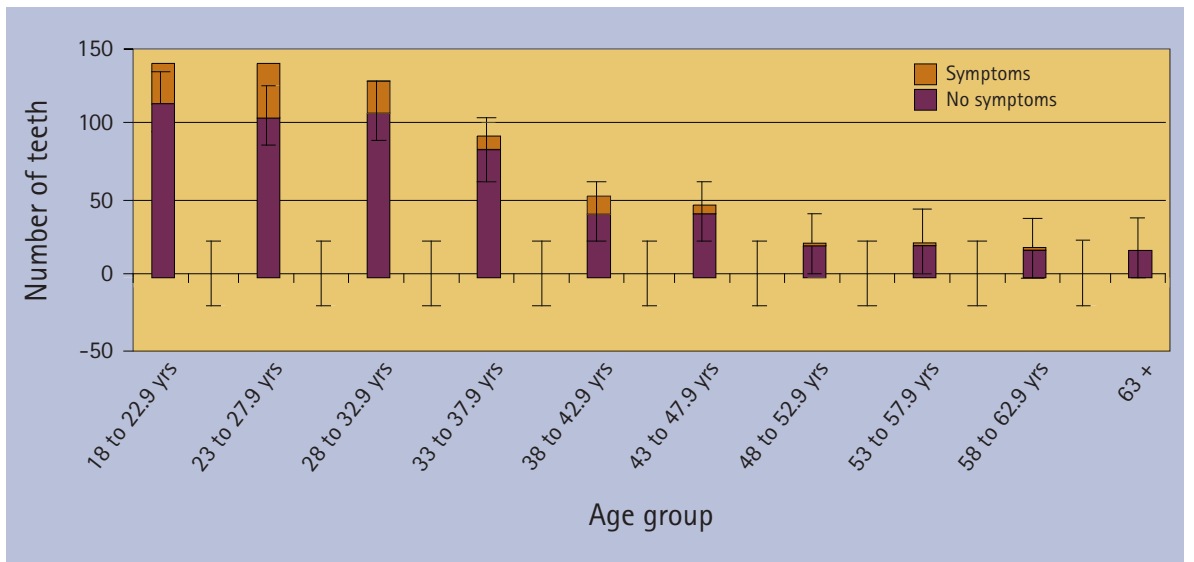

Fig. 1 Development of symptoms associated with impacted lower third molars in one year

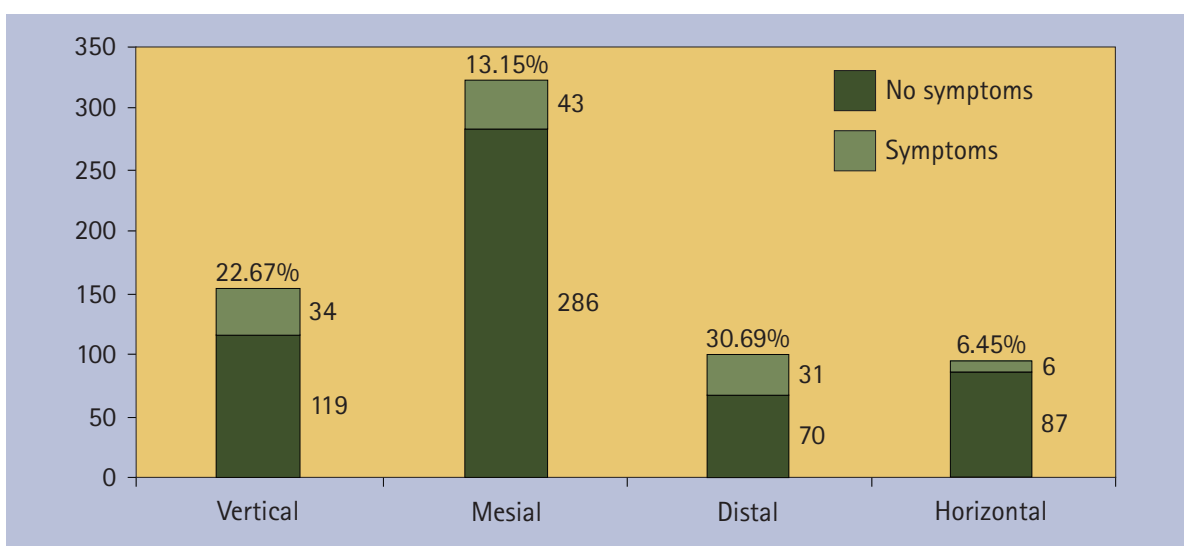

Fig. 2 Development of symptoms in 114 previously symptom-free impacted lower third molars after one year according to angulation

\section{Age and gender}

From the younger age group (18 to 34.99 years of age), 83 subjects (22.55\%) developed some form of symptoms. Out of the age group of 35 to 49.99 years of age, 28 subjects (20.89\%) developed symptoms in the year studied and similarly, for the older age group (50 years of age and above), only three subjects (5\%) developed symptoms in the study period. Figure 1 shows the distribution of the development of symptoms over one year in the sample according to age. Pearson chisquare tests showed that the inverse association between age and the development of symptoms in impacted wisdom teeth is statistically significant $(p=0.0028)$. There was no statistically significant difference in the development of symptoms between males and females.

\section{Angulation and degree of impaction}

When angulation of impaction was considered, the distally impacted lower third molar was associated more frequently with symptoms when compared to the other angulations. This association is statistically significant ( $p \leq 0.001$ ) and its distribution is shown in Figure 2.

When we look at symptoms as SIGN symptoms only (infection, severe pain and caries) and discard any other type of symptoms, the distally impacted lower third molar still had a higher incidence of symptoms in the one-year study $(24.69 \%$ of all distally-impacted third molars) when compared to the other angulations: vertical 10.29\%, mesial 5.48\% and horizontal $3.34 \%$. This distribution is also statistically significant ( $\mathrm{p} \leq 0.001)$.

23.05\% of all partially erupted teeth became symptomatic when compared to only $10.49 \%$ of the unerupted teeth. This difference was statistically significant ( $p \leq 0.001$ ) and is illustrated in Figure 3. When we only consider SIGN symptoms and disregard other symptoms, the distribution is still statistically significant ( $p=0.004)$.

This result was expected for two main reasons: firstly, the SIGN guidelines discourage the extraction of deeply impacted teeth and secondly, for reasons discussed 


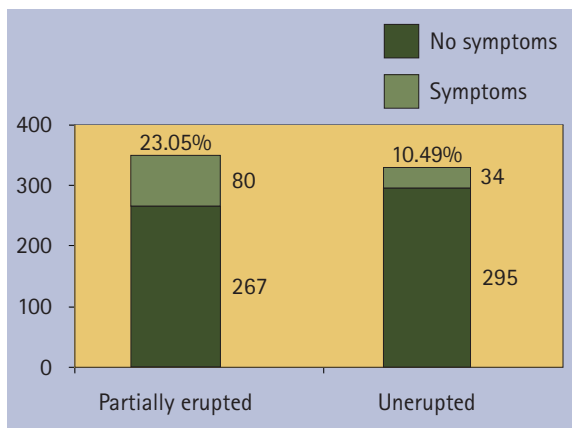

Fig. 3 Development of symptoms in 114 previously symptom-free impacted lower third molars after one year according to the degree of impaction

before, we could not irradiate patients for the purpose of this study and one could hypothesise that some of our unerupted teeth could be pathological but due to the short period of study (one year follow-up only), they were still asymptomatic. It is, however, imperative to note that there is a general consensus that unerupted teeth normally do not cause symptoms. These results could be explained by micro-communication of the impacted tooth with the oral cavity that is unable to be detected by a periodontal probe, or alternatively by recall bias.

There were no statistically significant differences between subjects who developed symptoms and those who did not develop symptoms for the average number of teeth, the maximum BPE scores, the average gingival bleeding index (MBI), and for the average mean plaque index (PI).

\section{Socio-demographic characteristics}

Student's $t$-tests showed that all but one possible socio-demographic association were not statistically significant. The association that was found to be significant was 'reason for last visit to the general dental practitioner (GDP)', where 23.52\% of subjects whose last visit to the GDP was prompted by a dental problem developed symptoms associated with their impacted lower third molar within the study period, whereas only $12.14 \%$ of those subjects whose last visit to their GDP was for a check-up or routine dental treatment developed some sort of symptom from their wisdom teeth. The $\mathrm{p}$ value is 0.041 for this association.

The data found to have no statistically significant associations (with their respective $p$ values) were: education after

Table 2 Development of symptoms within one-year according to Carstairs \& Morris deprivation categories

\begin{tabular}{|l|l|l|l|l|l|l|l|l|l|}
\multicolumn{2}{c|}{} & \multicolumn{6}{|c|}{ Deprivation category 1 (least deprived) to 7 (most deprived) } \\
\cline { 2 - 11 } & $\mathbf{1}$ & $\mathbf{2}$ & $\mathbf{3}$ & $\mathbf{4}$ & $\mathbf{5}$ & $\mathbf{6}$ & $\mathbf{7}$ & Total \\
\hline \multirow{3}{*}{$\begin{array}{l}\text { Symptoms } \\
\text { within } \\
\text { one year }\end{array}$} & No symptoms & 42 & 34 & 42 & 99 & 47 & 37 & 6 & 307 \\
\cline { 2 - 11 } & Symptoms & 12 & 10 & 19 & 10 & 12 & 37 & 14 & 114 \\
\cline { 2 - 11 } & Total & 54 & 44 & 61 & 109 & 59 & 74 & 20 & 421 \\
\hline
\end{tabular}

minimum school leaving age $(p=0.191)$, employment status $(p=0.560)$, frequency of brushing teeth $(\mathrm{p}=0.305)$, occasional use of mouthwashes $(p=0.116)$, occasional teeth flossing $(\mathrm{p}=0.124)$, frequency of dental appointments $(p=0.133)$, length of time since patient last visited the dentist ( $p=0.335)$, smoking $(p=0.291)$ and drinking 14 or more units of alcohol per week ( $p=0.447)$.

Similarly, there were no statistically significant associations between development of symptoms and deprivation category ( $\mathrm{p}$ $=0.058$ ) (Table 2).

\section{DISCUSSION}

Almost $70 \%$ of all patients attending the baseline appointment also attended the follow-up. This translated into just over 30\% attrition, which is remarkable for a dental primary care study. However, there were statistically significant differences between those patients who attended the one-year follow-up appointment $(n=421)$ and those who failed to attend this appointment ( $\mathrm{n}=$ 152). Many research studies are criticised for lacking in patients from more deprived backgrounds. This study had a 13\% failure rate in the most deprived category (7) contrasted with a 45\% failure rate in the least deprived category (1). There were not, however, statistically significant associations between development of symptoms and deprivation category (Table 2). This may imply that deprivation categories do not play a major role in the development of the symptoms studied; therefore, selection bias due to depcat scores might not have such an impact in the generalisability of the results of this study. The sample size in this study is too small to estimate separate risks for different socio-economic groups; future, larger scale studies would permit such an analysis, but the findings of this study must be viewed with caution in this regard.
Most participants in the study were below 35 years of age, and we acknowledge the lack of older patients in the study. There are a few probable reasons for this. One possible explanation is the fact that many 'older' patients were subjected to different, more interventionist, extraction policies. This cohort effect may have influenced the results of our study in the sense that many older patients could have had their wisdom teeth removed in the past and those who retained their impacted lower third molars were 'the best of the bunch'. This fact should also be considered when interpreting our results.

Over 30\% of all distally impacted teeth caused symptoms in the study period. This may prompt the profession to ask questions such as 'Should we start extracting more distally impacted mandibular third molars?' Alternatively, can we be even more audacious and dare to ask the question 'Should we extract distally impacted lower third molars prophylactically?' This is not an easy question to answer, as there is a conflict of interest between the three main parties involved in the discussion. The furst party is the clinician, whose main aim is to provide the best treatment for the patient. However, all outcomes are dependent on the interests of the second party involved in the discussion, the patient. The main interest of patients is presumably not having any pain, discomfort, physical or social disability, ${ }^{16}$ but what is worse for a patient? Having one, two or several episodes of pericoronitis for example, or going through the surgical extraction of an impacted mandibular third molar and its potential associated morbidity? This question cannot be answered generically, as the responses would vary quite significantly depending upon which person is asked and what previous pain experience the patient had. For example, one person experiencing two episodes of pericoronitis a year may 
find it unacceptable to retain the 'offending' tooth, whereas another person in the same circumstances may not even consider having the wisdom tooth removed. Health experiences and personality may play a major role in determining the best course of action for different patients. Knowledge of the incidence of symptoms is of paramount importance for the clinician, who can then (together with the patient) decide on the best treatment plan, as patients' preferences may differ from those of dentists. A study that compared patients' outcome preferences for removal and retention of mandibular third molars in Sweden and Wales found that the outcomes of surgery were considered worse after third molar removal than retention. ${ }^{17}$ In another study where patients had a third molar removed, more patients found that the effect on intake and enjoyment of food was a more adverse event than pain following surgery. ${ }^{18}$

In the distally impacted lower third molar issue we must remember that there is a chance that some impacted lower third molars which are mesially or vertically impacted will change their position to a disto-angular position. ${ }^{19}$ This fact could exacerbate the problem in future as distally impacted lower wisdom teeth are perceived to be more difficult to remove than vertically or mesially impacted wisdom teeth.

Finally, the third party involved in this discussion is the one formed by health managers. Because resources are always scarce, health managers have to find a way to best allocate them. The "watchful monitoring' policy seems to be favoured by health managers as in the short term, it seems to be a less costly procedure when compared to extractions and surgical extractions. In 1999 Edwards et al. ${ }^{20}$ found that it is more cost-effective to retain an impacted lower third molar than to remove it, but they also found that it becomes more cost-effective to remove an impacted lower third molar if pericoronitis, caries etc develop. Despite this conclusion, the Cochrane Oral Health Group systematic review conducted in 2005 found no evidence to support or to refute the prophylactic removal of impacted wisdom teeth. ${ }^{7}$ It would be ideal if health managers (and the clinician!) could somehow know exactly which teeth will cause problems in future. Therefore the suggestion from this study is that the distally impacted lower wisdom tooth and those teeth which are partially erupted are more likely to be associated with symptoms and should be watched more closely.

The fact that more patients with partially erupted teeth developed more symptoms compared to patients with unerupted teeth came as no surprise, as the main outcomes considered in the study were pericoronitis, infection, pain and discomfort (which are more common in partially erupted teeth due to their communication with bacteria from the oral environment). This fact has also been documented in the literature. ${ }^{21}$

It is noteworthy that $10.49 \%$ of all unerupted teeth caused symptoms in our sample. Unerupted teeth are generally considered not to cause symptoms by clinicians. There are a few possible explanations for this occurrence in our study. The most reasonable is 'recall bias', where patients responded positively to the question 'Have you had pain in your wisdom tooth/teeth in the past year?' but in fact had no symptoms at all, or perhaps those symptoms could have been caused by another tooth and the patient erroneously mistook it as being caused by the third molar. It can be hypothesised that these $10.49 \%$ of unerupted teeth which are reported as causing symptoms are in fact erupting and have become partially erupted and in communication with the oral cavity. However, the examiner did check the distal of the lower second molar with a periodontal probe and all teeth which were in communication with the oral cavity were considered partially erupted and not unerupted.

There is evidence in the literature that the presence of erupted or partially erupted third molars can increase one's level of periodontal disease. ${ }^{22,23}$ A recent study looking at risk markers for periodontal pathology reported that a probing depth of $4 \mathrm{~mm}$ or more in the third molar region can be predictive of detection of periodontal pathology. ${ }^{24}$ Although this evidence seems to suggest that the presence of a third molar by itself can increase the risk of periodontal pathology, our findings suggested that there is no association between established periodontal pathology (as measured by the parameters and indexes used in our study) and the development of symptoms associated with impacted lower third molars in the sample. Further research, including bacteriological analysis for instance, would be useful to determine if there is any causal correlation between periodontal disease and infection around the operculum in third molars.

Asymptomatic approximal caries were not included in our analysis due to the ethical implications of irradiating patients for a research study. This implies that our results might underestimate 'symptoms', as some teeth in our sample could have developed visually undetectable approximal caries, but this was not an outcome intended to be measured in this study.

The frequency of toothbrushing was also recorded (it is of note that this was the reported frequency of brushing and despite patients receiving a verbal explanation from the study's dental nurse and all questionnaires being anonymous, it is believed that there was still a small percentage of subjects who tried to present themselves in a better light and responded to the questionnaire with a higher frequency of brushing than was actually the case). The duration of brushing or the brushing technique used were not asked and patients were not asked what type of toothbrush they used, as the intention of the questionnaire was not to measure oral hygiene but attitudes towards dental health. The same can be said for the frequency of flossing and the use of a mouthwash. Therefore, in interpreting the results for these variables one should take these observations into consideration.

Out of all the socio-economic variables, the only one found to have an association with the development of the symptoms studied was the 'reason for the last visit to the dentist', where patients whose last dental visit was prompted by a dental emergency unrelated to the wisdom tooth reported more symptoms than those patients whose last visit to the dentist was due to routine dental treatment.

The fate of an impacted lower third molar is still in large part a mystery. However, the results of this study show that there is clearly a definite association between the degree and the angulation of impaction and the development of symptoms observed. This study sheds new light on the occurrence of symptoms in association with impacted lower third molars. 
We now know that the development of symptoms associated with these teeth is no longer completely due to chance. Future studies should focus on symptoms and signs (including radiographic evidence) and pathology associated with impaction over a long period of observation. Results should be analysed according to the tooth's angulation and degree of impaction.

The development of this study was supported by The Wellcome Trust (061636/HS/SH/MW/sf). The project also had collaboration from the primary care research networks TayRen (Tayside Primary Care Research Network), FResCo (Fife Research Cooperative) and Westnet (West of Scotland Research Network) and its dental branch GRID (Glasgow Research Initiative in Dental Primary Care). Professor Pitts acknowledges support from the Chief Scientist Office, which core funds the Dental Health Services Research Unit. The authors would like to acknowledge the collaboration and assistance of Professor Lorna MacPherson at the Department of Dental Public Health at Glasgow Dental School. The views expressed in this paper are those of the authors and may not be shared by the funding bodies.

1. Landes D P. The relationship between dental health and variations in the level of third molar removals experienced by populations. Community Dent Health 1998: 15: 67-71.

2. Thomas D, Walker R, Smith A, Shepherd J. The provision of oral surgery services in England and Wales 1984-1991. Br Dent J 1994;
176: 215-219.

3. Worrall S F, Riden K, Haskell R, Corrigan A M. UK National Third Molar project: the initial report. Br J Oral Maxillofac Surg 1998; 36: 14-18.

4. NICE. Guidance on the removal of wisdom teeth. York: National Institute for Clinical Excellence, 2000

5. SIGN. Management of unerupted and impacted third molar teeth. Edinburgh: Scottish Intercollegiate Guidelines Network, 2000.

6. Tilley C, Crawford F, McCann M, Clarkson J, Pitts $\mathrm{N}$. Confounding in the analysis of NICE guideline implementation. bmi.com, 2 December 2004. http:// www.bmj.com/cgi/eletters/329/7473/999\#87787 (accessed 17 January 2009). .

7. Mettes T G, Nienhuijs M E L, van der Sanden W J M Verdonschot $\mathrm{E} H$, Plasschaert A J M. Interventions for treating asymptomatic impacted wisdom teeth in adolescents and adults. Cochrane Database Syst Rev 2005; (2): CD003879.

8. Mercier P, Precious D. Risks and benefits of removal of impacted third molars. A critical review of the literature. J Oral Maxillofac Surg 1992; 21: 17-27.

9. Flick W G. The third molar controversy: framing the controversy as a public health policy issue. J Oral Maxillofac Surg 1999; 57: 438-444.

10. Ogden G R. Removal of unerupted teeth. In Pedlar J, Frame J W (eds) Oral and maxillofacial surgery: an objective-based textbook. 2nd ed. pp 45-66. London: Churchill Livingstone, 2007.

11. Ainamo J, Barmes D, Beagrie G, Cutress T, Martin J, Sardo-Infirri J. Development of the World Health Organization (WHO) community periodontal index of treatment needs (CPITN). Int Dent J 1982; 32: 281-291.

12. Ainamo J, Bay I. Problems and proposals for recording gingivitis and plaque. Int Dent J 1975; 25: 229-235.

13. Greene J C, Vermillion J R. The Oral Hygiene Index: a method for classifying oral hygiene status. J Am
Dent Assoc 1960; 61: 172-179.

14. Carstairs V, Morris R. Deprivation and health in Scotland. Aberdeen: Aberdeen University Press, 1991.

15. Fernandes M J, Ruta D A, Ogden G R, Pitts N B, Ogston S A. Assessing oral health-related quality of life in general dental practice in Scotland: validation of the OHIP-14. Community Dent Oral Epidemiol 2006; 34: 53-62.

16. Locker D. Measuring oral health: a conceptual framework. Community Dent Health 1988; 5: 3-18.

17. Liedholm R, Knutsson K, Lysell L, Rohlin M, Brickley $M$, Shepherd J. Third molar treatment outcome: a comparison of patients' preferences in Sweden and Wales. Br Dent J 2005; 199: 287-291.

18. Ruta D A, Bissias E, Ogston S, Ogden G R. Assessing health outcomes after extraction of third molars: the postoperative symptom severity (PoSSe) scale. Br J Oral Maxillofac Surg 2000; 38: 480-487.

19. Swerin I, van Wowern N. A radiographic 4-year follow up study of asymptomatic mandibular third molars in young adults. Int Dent J 1990; 40: 24-30.

20. Edwards M J, Brickley M R, Goodey R D, Shepherd $J$ P. The cost, effectiveness and cost effectiveness of removal and retention of asymptomatic, disease free third molars. Br Dent J 1999; 187: 380-384.

21. Peterson L J, Ellis E, Hupp J R, Tucker M R. Contemporary oral and maxillofacial surgery. Philadelphia: Mosby, 2003.

22. Moss K L, Serlo A D, Offenbacher S, Beck J D, Mauriello S M, White R P Jr. The oral and systemic impact of third molar periodontal pathology. J Oral Maxillofac Surg 2007; 65: 1739-1745.

23. Moss K L, Beck J D, Mauriello S M, Offenbacher S, White R P Jr. Third molar periodontal pathology and caries in senior adults. J Oral Maxillofac Surg 2007; 65: 103-108.

24. White R P Jr, Phillips C, Hull D J, Offenbacher $S$, Blakey $\mathrm{G} \mathrm{H}$, Haug R M. Risk markers for periodontal pathology over time in the molar and non-third molar regions in young adults. J Oral Maxillofac Surg 2008; 66: 749-754. 